\title{
DIRECTORIO DE PROFESORES VINCULADOS A LA NARRATIVA AUDIOVISUAL DE ESPAÑA Y AMÉRICA LATINA
}

Francisco Javier López Rodríguez (Universidad de Granada)

http://dx.doi.org/10.12795/AdMIRA.2009.01.27

\begin{tabular}{|c|c|c|}
\hline NOMBRE & UNIVERSIDAD & PAIS \\
\hline Gabriel Alba Gutiérrez & Universidad Nacional de Colombia & Colombia \\
\hline María Aparecida Moura & Universidade Federal de Minas Gerais & Brasil \\
\hline José Vicente Arismendi & Pontificia Universidad Javeriana- Cali & Colombia \\
\hline $\begin{array}{l}\text { Esperanza Astroz } \\
\text { Avellaneda }\end{array}$ & Pontificia Universidad Javeriana- Cali & Colombia \\
\hline $\begin{array}{l}\text { Xavier Berenguer } \\
\text { Vilaseca }\end{array}$ & Iniversitat Pompeu Farra & España \\
\hline Augusto Bernal Jiménez & Escuela de Cine Black María & Colombia \\
\hline Diego Bonilla Castañeda & Universidad de Syracuse & $\begin{array}{l}\text { Estados } \\
\text { Unidos }\end{array}$ \\
\hline Roger Bundt & Universidade Santa Cruz Do Sul & Brasil \\
\hline Alfredo Caminos & $\begin{array}{l}\text { Universidad de Córdoba, Universidad de } \\
\text { Barcelona }\end{array}$ & Argentina \\
\hline Angel Pablo Cano Gómez & Universidad Católica de Murcia & España \\
\hline $\begin{array}{c}\text { Concepción Cascajosa } \\
\text { Virinos } \\
\end{array}$ & Universidad Carlos III & España \\
\hline $\begin{array}{l}\text { Maritza Ceballos } \\
\text { Saavedra }\end{array}$ & Pontificia Universidad Javeriana & Colombia \\
\hline René Chargoy & Universidad del Mar & México \\
\hline Antonio Checa Godoy & Universidad de Sevilla & España \\
\hline Ernesto Correa Herrera & Universidad de Medellín & Colombia \\
\hline Mauricio Durán Castro & Pontificia Universidad Javeriana & Colombia \\
\hline $\begin{array}{l}\text { Luis Orlando Espinosa } \\
\text { Ramírez }\end{array}$ & Universidad Nacional de Colombia & Colombia \\
\hline Gabriela Fabbro & Universidad Austral & Argentina \\
\hline Sergio Ferreira Do Amaral & Universidade Estadual de Campinas & Brasil \\
\hline $\begin{array}{l}\text { Elena María Galán } \\
\text { Fajardo } \\
\end{array}$ & Universidad Carlos III & España \\
\hline Miguel Angel Garnica & UNAM & México \\
\hline Ruggero Garofalo & Universidad Intercontinental & México \\
\hline $\begin{array}{l}\text { Rodolfo Goncalves } \\
\text { Araujo }\end{array}$ & PUC-SP & Brasil \\
\hline Inmaculada Gordillo & Universidad de Sevilla & España \\
\hline Vicente Gosciola & PUC-SP & Brasil \\
\hline Virginia Guarinos & Universidad de Sevilla & España \\
\hline Alma Elena Gutiérrez & $\begin{array}{c}\text { Tecnológico de Monterrey- Campus } \\
\text { Monterrey }\end{array}$ & México \\
\hline
\end{tabular}




\begin{tabular}{|c|c|c|}
\hline Manel Jiménez Morales & Universitat Pompeu Fabra & España \\
\hline Jesús Jiménez Varea & Universidad de Sevilla & España \\
\hline Andrés Jurado Vélez & Pontificia Universidad Javeriana & Colombia \\
\hline Adriane Harder & Universidade de Sao Paulo & Brasil \\
\hline $\begin{array}{l}\text { Pedro Antonio Hellín } \\
\text { Ortuño }\end{array}$ & Universidad de Sevilla, U. de Murcia & España \\
\hline Alvaro Herrera & Universidad Santo Tomás & Colombia \\
\hline Pedro Lapera & Universidad Federal Fluminense & Brasil \\
\hline Julio César Lobo & Universidade Federal de Bahia & Brasil \\
\hline José Luis López Aguirre & Universidad Panamericana & México \\
\hline $\begin{array}{l}\text { Ana María López } \\
\text { Carmona }\end{array}$ & Universidad de Chile & Chile \\
\hline $\begin{array}{l}\text { Carlos Alfonso López } \\
\text { Lizarazo }\end{array}$ & Universidad de Medellín & Colombia \\
\hline $\begin{array}{l}\text { Francisco J. López } \\
\text { Rodríguez }\end{array}$ & Universidad de Sevilla & España \\
\hline $\begin{array}{l}\text { Ada Cristina Machado } \\
\text { Silveira }\end{array}$ & Universidade Federal de Santa Maria & Brasil \\
\hline Francisco Martín & Universidad de Málaga & España \\
\hline María José Martínez & Universidad Técnica Particular de Loja & Ecuador \\
\hline Miguel Angel Martínez & Universidad Católica de Murcia & España \\
\hline Pedro Matute Villaseñor & Universidad de Guadalajara & México \\
\hline Maria Luisa Mendonca & Universidade Federal de Goiás & Brasil \\
\hline Catalina Mier & Universidad Técnica Particular de Loja & Ecuador \\
\hline Maria Claudia Oliveira & Multirio & Brasil \\
\hline Jhon Jaime Osorio Osorio & Universidad de Medellín & Colombia \\
\hline $\begin{array}{l}\text { Miguel Ángel Pérez } \\
\text { Gómez }\end{array}$ & Universidad de Sevilla & España \\
\hline Xavier Pérez Torío & Universitat Pompeu Fabra & España \\
\hline Cristina del Pino Romero & Universidad Carlos III & España \\
\hline Ivan Pintor Iranzo & Universitat Pompeu Farra & España \\
\hline Denis Porto Renó & $\begin{array}{l}\text { Unicoc, Universidade Metodista de Sao } \\
\text { Paolo }\end{array}$ & Brasil \\
\hline $\begin{array}{l}\text { Inmaculada Postigo } \\
\text { Gómez }\end{array}$ & Universidad de Málaga & España \\
\hline Orlando Puente Moreno & Universidad Autónoma de Occidente & Colombia \\
\hline $\begin{array}{l}\text { María del Mar Ramírez } \\
\text { Alvarado }\end{array}$ & Universidad de Sevilla & España \\
\hline Omar Rincón & Pontificia Universidad Javeriana & Colombia \\
\hline Mayra I. Rivera & Universidad de Puerto Rico & Puerto Rico \\
\hline $\begin{array}{c}\text { Jerónimo León Rivera } \\
\text { Betancur }\end{array}$ & Universidad de la Sabana & Colombia \\
\hline Adriana Rodríguez & Pontificia Universidad Javeriana- Cali & Colombia \\
\hline Isabel Rodríguez Martínez & Universidad Central & Colombia \\
\hline Sandra Ruiz Moreno & Universidad del Rosario & Colombia \\
\hline María Jesús Ruiz Muñoz & Universidad de Málaga & España \\
\hline Fernando Saad & Universidad de Córdoba & Argentina \\
\hline Glòria Salvadó Corretger & Universitat Pompeu Farra & España \\
\hline
\end{tabular}




\begin{tabular}{|c|c|c|}
\hline Alan Salvadó Romero & Universitat Pompeu Farra & España \\
\hline $\begin{array}{c}\text { Jacqueline Sánchez } \\
\text { Carrero }\end{array}$ & Universidad de Sevilla & España \\
\hline Josefina Sánchez Martínez & Universidad Católica de Murcia & España \\
\hline Yamile Sandoval Romero & Universidad de la Sabana & Colombia \\
\hline $\begin{array}{c}\text { Fátima de los Santos } \\
\text { Romero }\end{array}$ & Universidad de Sevilla & España \\
\hline Ana María Sedeño & Universidad de Málaga & España \\
\hline J. Adolfo Soto & Universidad Autónoma de Baja & México \\
\hline $\begin{array}{c}\text { Tais Rios Salomao de } \\
\text { Souza }\end{array}$ & Universidad Metodista de Sao Paulo & Brasil \\
\hline Paula Trujillo & Pontificia Universidad Javeriana- Cali & Colombia \\
\hline Enrique Uribe Jongbloed & Universidad de la Sabana & Colombia \\
\hline $\begin{array}{c}\text { Norman Vélasquez } \\
\text { Álvarez }\end{array}$ & Universidad de Medellín & Colombia \\
\hline $\begin{array}{c}\text { Juan Mauricio Vélez } \\
\text { Lotero }\end{array}$ & Universidad de Medellín & Colombia \\
\hline Mauricio Vergara Hurtado & Pontificia Universidad Javeriana- Cali & Colombia \\
\hline Lucía Victoria Torres & Universidad Pontificia Bolivariana & Colombia \\
\hline Elizabeth Vidal & Universidad de Córdoba & Argentina \\
\hline Adriana Villamizar & Pontificia Universidad Javeriana- Cali & Colombia \\
\hline Adán Zamarripa Salas & UNAM & México \\
\hline José Francisco Zegers & Universidad de los Andes & Chile \\
\hline
\end{tabular}

